\title{
L'agglomération d'activités d'économie sociale: Mondragon, Montréal et la Toscane
} The agglomeration of the social economy activities: Mondragon, Montreal and Toscane A aglomeração das atividades da economia social: Mondragon, Montréal e da Toscane La aglomeración de las actividades de la economía social: Mondragon, Montreal e la Toscane

\author{
Jacques Prades*
}

Recebido em 19/12/2006; revisado e aprovado em 16/3/2006; aceito em 23/6/2006.

\begin{abstract}
Résumé: L'objet de cet article est de relater les résultats de trois expériences d'agglomérations d'activités d'économie sociale au Pays basque espagnol, en Italie et à Montréal. L'étude a nécessité sept voyages au cours duquel ont été réalisés des enquêtes de terrain et des entretiens ${ }^{1}$. Elle vise à énoncer un certain nombre de récurrences observées après avoir décrit succintement les terrains. Dans une première partie, nous énoncerons quelques préalables méthodologiques, définitions et hypothèses. Dans une deuxième partie, nous ferons une description des trois expériences. En conclusion, nous regrouperons les ingrédients communs à ces trois expériences pour en tirer quelques conséquences analytiques. Mots-clé: Agglomération; économie sociale; expériences.

Abstract: The objective of this article is to give an account about results of three agglomeration experiences about the social economy activities in spanish Bilbao country, in Italy and Montreal. The study needee seven travels around the places where the informations were picked about the grounds and the expenses. It has as objective to express an amount of observed resorts after the succinct description about the grounds. In a first side, we will express some methodologic prelinimaries, definitions and hyphotesis. In a second side, we will make a description of the three experiences. For conclusion, we will group the common ingredients of there three experiences to take some analytic consequences.
\end{abstract}

Key words: Agglomeration; social economy; experiences.

Resumo: O objeto desse artigo é de relatar os resultados de três experiências de agrupamentos de atividades de economia social no País basco espanhol, na Itália e em Montreal. Para o estudo foram necessárias sete viagens durante as quais foram realizadas pesquisas de campo e entrevistas. Procura-se enunciar certo número de recorrências observadas, após terem sidos descritos sucintamente os terrenos. Numa primeira parte enunciaram-se alguns princípios metodológicos, definições e hipóteses. Numa segunda parte far-se-á uma descrição das três experiências. Como conclusão, reagrupar-se-á os ingredientes comuns a essas três experiências, para se obter algumas conseqüências analíticas.

Palavras-chave: Aglomeração; economia social; experiências.

Resumen: El objetivo de este artículo es relatar los resultados de las trés experiéncias de las aglomeraciones de las actividades de la economía social al País Basco español, en Itália y Montreal. El estudio tuvo la necesidad de siete viajes a los sítios dónde fueron recoagidas las informaciones sobre los terrenos y los gastos. Tiene como objetivo expresar una cierta caritidad de recurréncias observadas después de haber descrito de manera sucinta los terrenos. En la primera parte, nosotros expresaremos algunas previas metodológicas, definiciones e hipótesis. En la segunda parte, nosotros haremos una descripción de las trés experiencias. Para efecto de conclusión, nosotros reagruparemos los ingredientes comunes às estas trés experiencias para sacar algunas consecuencias analíticas.

Palabras clave: Aglomeración; economía social; experiencias.

\section{Première partie Quelques repères méthodologiques}

Nous avons choisi l'analyse de deux expériences coopératives et une expérience associative.

Le premier cas est celui de Mondragon. Situé dans le pays basque espagnol, le complexe coopératif de Mondragon regroupe plus de 116 coopératives. Créé en 1956, le complexe a vécu l'histoire industrielle de ces trente dernières années sans jamais licencier car le droit de licenciement est interdit, avec un éventail des salaires qui est resté longtemps compris en- tre 1 et 3. Le cas de l'Italie est un peu différent car le nombre de coopératives est un des plus important d'Europe et la succession des formes coopératives est un trait essentiel de l'économie italienne. Nous nous concentrons ici sur l'histoire de la Banca Etica pour caractériser ce mouvement. Particulièrement présente en Toscane, elles représentent une émanation de la société civile.. Le troisième cas est celui des CDEC montréalaises qui sont de véritables comités de quartier mais à l'échelle d'un arrondissement qui gèrent la ville de Montréal.

Le choix de ces terrains a été guidé par deux considérations :

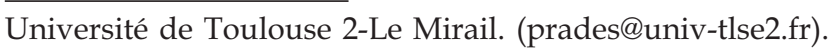


- une première raison est que les initiatives qui nous intéressent ici se situent autour d'une dynamique qui emprunte trois voies étroitement liée mais dont l'une prédomine : celle de l'entrepreneuriat collectif comme à Mondragon, celle de la société civile comme au Québec et celle d'une substitution partielle de 1'Etat comme en Italie. En devenant exclusif au détriment des autres, chaque pôle présente un risque de dilution de l'originalité de l'initiative. En effet, le premier pôle tire vers la banalisation des marchés, le second pôle pousse au risque de communautarisme et le troisième pôle vers le risque d'une instrumentalisation, voire d'une bureaucratisation. Chacun des pôles est donc en tension avec les autres mais avec des dominantes.

- une deuxième raison est que nous ne voulions pas nous enfermer dans un débat franco-français de l'économie sociale et l'économie solidaire en évitant les deux pièges de ce débat : soit nier leur différence soit creuser un gouffre entre les deux perspectives. Le contournement de ce débat a consisté à étudier des expériences étrangères et à ne revenir sur la France qu'à la fin de l'étude des terrains. Le concept «d'économie solidaire » peut utilement servir à identifier certaines structures qui ne sont pas mises en relief autrement. Mais il s'avère fragile et ne semble pas faire l'unanimité ni d'un point de vue théorique ni du point de vue des praticiens, ce qui expliquerait sa lenteur de diffusion. Un concept ne s'impose que s'il résulte de la convergence d'un mouvement descendant de chercheurs, technocrates, etc. et d'un mouvement $\mathrm{du}$ bas qui par ces pratiques se reconnaît dans la conceptualisation. Nous avons donc préféré opté pour $\mathrm{l}^{\prime}$ expression $\mathrm{d}^{\prime}$ " entreprises sociales» et $\mathrm{d}^{\prime}$ «initiatives solidaire ».

\section{Deux mobiles historiques récurrents}

Ces histoires respectives font apparaître des récurrences qui obéissent à deux mobiles parfois dissociés :

- Une première logique est celle de la nécessité. Des populations en marge cherchent à construire des parcours individuels et collectifs en reconquérant des droits dont ils avaient été exclus : droit au travail, droit au logement, droit au crédit. H. Desroches avait l'habitude de dire que ces pratiques sociales étaient «filles de la nécessité ».

- Une deuxième logique est celle de la revendication d'une identité ou d'un destin commun. La population d'un territoire se rassemble en vue d'un objectif commun : l'emploi par le développement local, culturel, environnemental ou éducatif.

Les deux logiques peuvent se regrouper bien qu'il puisse y avoir entre elles un conflit de prévalence identitaire : est-ce que ce qui fait « identité », c'est la classe (les exclus, les pauvres, etc.) ou le territoire (c'est-à-dire la frontière qui sépare le dedans du dehors) ou les deux à la fois?

\section{Des expériences agglomérées}

Dans ce travail, nous ne cherchons pas à grossir des micro-activités exemplaires qui pourraient servir de "prêts à porter ». Nous étudions des entreprises sociales et des initiatives solidaires dont la longétivité témoigne d'une possibilité effective pour un groupe social de produire, consommer et répartir et échanger autrement. C'est dire que ce qui nous intéresse, ce sont les structures coopératives et non la coopération. En effet, dans le format néo-classique, les économistes découvrent que sous certaines conditions, «la coopération » est préférable à la concurrence. Mais la coopération qu'ils évoquent est celle qui favorise en dernier ressort l'intérêt individuel. Alors que dans les coopératives que nous évoquons, nous venons de l'indiquer, on part du principe inverse selon lequel l'intérêt individuel doit être dépassé. Il y a bien également un point de vue normatif dans ce travail, largement explicité mais il a la différence des néoclassiques, nous ne construisons pas de modèle sur une conception théorique non explicitée et idéelle (le marché). Au contraire, c'est sur des réalités empiriques que nous valorisons les hypothèses sur lesquelles elles reposent. Plus fortement, ce travail tente de faire ressurgir explicitement la mémoire collective de toutes les formes d'autodétermination que tous les pouvoirs (économiques mais aussi et surtout politiques) contribuent à étouffer. 
Des formes non-exclusives

Un des objectifs poursuivis est de montrer qu'après avoir considéré en France le modèle coopératif comme la référence de l'économie sociale jusqu'à la fin des années soixante (et même un peu plus tard avec C. Vienney), on en est venu à partir des années quatre vingt à ne rechercher de la nouveauté que dans certaines associations (ce que l'on nomme en France "l'économie solidaire»). Or, nous cherchons à montrer que dans les processus d'agglomération qui sont les seuls à nous intéresser ici, les deux formes ne sont pas exclusives l'une de l'autre mais qu'elles se complètent. Davantage encore, la forme associative à la française, entraîne presque inévitablement une usurpation de pouvoir considérable par son président ou son directeur (en raison des différences d'objectifs entre administrateurs et directeur), en même temps qu'elle plonge les structures dans une dépendance excessive à l'égard des politiques publiques (du fait de l'instrumentalisation liée à la nécessité de ressources publiques) ou encore qu'elles tendent à s'éloigner des populations locales qui les ont très souvent engendrés (rigidification des structures). Les coopératives peuvent être un outil de sociations politiques à but économique.

Des entrées diverses

Le deuxième objectif est de montrer qu'aucune entrée en matière d'agglomération d'activités n'est privilégiée : on peut débuter par des coopératives de production comme le complexe de Mondragon dans le pays basque espagnol, de consommation comme les districts italiens dans leur composante coopérative dans la troisième Italie ou une forme de démocratie comme les Corporations de Développement Economique Communautaire de Montréal au Québec, plus communément appelées les CDEC qui sont des associations qui ont à voir avec les coopératives d'habitation. L'économie sociale prend du sens lorsqu'elle émane d'initiatives locales et qu'en grossissant, elle ne perd pas de vue son fondement et lorsqu'elle s'avère être plus performante et plus équitable dans tous les segments où elle intervient. C'est là qu'elle peut être le vecteur d'une pédagogie collective pour la transformation sociale.

\section{Des mobiles politiques}

Le troisième objectif de cette étude est de montrer que les coopératives ne sont pas seulement des entreprises comme les autres qui répartissent autrement. Car le mobile qui réunit les expériences que nous évoquons ici est politique au sens où elles n'attendent pas d'un Etat affaibli les clés d'une nouvelle régulation sociale. Elles cherchent par des voies associées à prendre elle-même leurs destins en main. Cette thèse est allée souvent à l'encontre des congrès de la coopération ${ }^{2}$ qui en défendant le principe de neutralité n'ont pas brillé de clarté sous l'épisode Vichyssois. Tous les acteurs des initiatives présentées ici ont compris qu'on assistait à une redéfinition de l'Etat providence et qu'une attitude de crispation gelait les initiatives émergentes. Cette impossibilité de remettre en cause l'Etat en France, autrement que sous sa forme libérale, est probablement une des clés de la difficulté d'émergence $d$ 'une nouvelle économie sociale.

\section{Deuxième partie Etudes de cas}

Mondragon

Mondragón est une ville de 25000 habitants, située au pied de l'Udalaitz, à 30 $\mathrm{km}$ au sud-est de Guernica et au nord-est de Vitoria, entre Bilbao $(50 \mathrm{~km})$ et Saint Sébastien. d'herbe. En octobre 1955, pour contourner les autorisations de création et d'implantation d'entreprises contrôlées par l'Etat, cinq jeunes fondateurs eurent l'idée de reprendre une entreprise en difficulté de produits électriques et mécaniques à usage domestique qui se trouvaient à Vitoria. C'est ainsi que naît la première entreprise coopérative ULGOR.

Ce n'est qu'un an après la reprise, en avril 1956, que les fondateurs transférèrent l'entreprise à $30 \mathrm{Km}$, dans le petit village de Mondragón. Il est fort probable que la présence d'un jeune prêtre activiste, Don José María Arizmendiarrieta, soit à l'origine de 
ce déplacement géographique.

Le prêtre est en effet arrivé à Mondragón en 1941 et il n'en bougera pas jusqu'à sa mort en 1976. Mondragón est issu $\mathrm{du}$ mouvement social catholique. J-M Ormaetxea, un des seuls compagnons à être toujours vivant ${ }^{3}$.prétend que le mobile de $l^{\prime}$ expérience tient à l'équité d'origine chrétienne qui sous-tend le processus : lutte contre la pauvreté au début de l'expérience puis lutte contre la division sociale et la répartition des revenus ${ }^{4}$. Comme le pouvoir franquiste était dictatorial et totalitaire, il n'y avait pas d'action politique ni d'action syndicale de la part des ouvriers de sorte que le prête Don José Maria ne faisait que défendre "une cause avec espoir », un recours à la communauté.

$\mathrm{C}^{\prime}$ est ce contexte politique essentiel qui permet d'expliquer la naissance du complexe de Mondragón en deux grandes périodes économiques à partir de laquelle on peut comprendre la période actuelle.

1956-1970 L'engagement chrétien réformiste dans la construction d'un complexe gravitaire

C'est en 1943 qu'est créée une école professionnelle à Mondragón car la permanence de la formation est un trait significatif de l'expérience historique des coopératives: "Pour démocratiser le pouvoir, il faut socialiser le savoir » disait le curé rouge, ajoutant que « le futur était dans la vertu d'une action éducative d'envergure et $\mathrm{d}^{\prime}$ un processus associatif qui intègre tous les hommes comme des frères » (Mondragón Al Dia Cara Al Futuro). C'est de cette école que sont sortis les cinq premiers coopérateurs, âgés de 29 à 32 ans, qui créèrent, fin 1955, Ulgor spécialisé à l'époque dans le petit matériel de chauffage à pétrole dont les statuts ne seront véritablement votés et légalement créés qu'en 1959.

Durant cette période, Arrasatemachinerie s'installe à Mondragón en 1957 et Ulgor crée une division électronique. Ces deux coopératives tractent la création des autres coopératives qui gravitent autour d'elles.

En 1958, les salariés associés sont exclus du système général de la Sécurité Sociale, par ordre du Ministère du travail. Comme les associés ne sont ni des salariés ni des patrons, ils n'ont pas droit à la sécurité sociale traditionnelle. Est ainsi créé Lagun Aro, un organisme de prestations sociales spécifiques qui remplit les deux rôles de la sécurité sociale française.

En 1959, la Caja Laboral Popular (société coopérative de crédit) voit le jour sous l'impulsion de Don José Maria, car le développement coopératif nécessite des financements. Il s'agit d'une caisse dont l'épargne provient des "mamies ", selon l'expression d'Arizmendiarrieta. Il est fort probable que la confiance que suscite le curé auprès des personnes âgées a joué un rôle significatif dans la collecte de l'épargne. La caisse a pour mission de financer les petites coopératives de Mondragón, Ulgor, Arrasate, San José, etc- qui n'intéressent pas les institutions bancaires traditionnelles.

Le fonctionnement des coopératives peut être décrit de la manière suivante :

Soit un travailleur-associé qui perçoit « une avance sur travail » ${ }^{5}$ de $300 €$ par mois en $1970^{6}$. Le financement au départ est le suivant :

- apport du travailleur-associé (los socios), environ 10 mois d'avance, soit $3000 €$ (crédit de la Caja Laboral ou déduction mensuelle). Cet apport est définitivement acquis par la coopérative et donc perdu pour les socios, et ne leur rapporte pas d'intérêt à hauteur de $25 \%$ de l'apport (75\% donne lieu à intérêts percevables au départ du socios).

- Aide de l'Etat (pour création d'emploi): $6000 €$

- Apport en prêt de la Caja Laboral: $21000 €$

Au total, le poste coûte sur l'année 30 000 euros (et l'apport de l'associé est de 10 $\%$ contre le double en 1956).

Sur les bénéfices réalisés par la société en fin d'année, l'associé touche un intérêt (hors les $25 \%$ de l'apport initial) qui constitue un dépôt dans la coopérative qui lui sera « ristourné » en cas de départ (personnel ou à la retraite). C'est donc une forme d'épargne salariale.

La mise en réserve des excédents est donc un puissant vecteur d'autofinancement.

Reste une difficulté : l'apport qui doit être fait au départ. Les premiers fondateurs n'avaient pas les sommes disponibles. Il a donc fallu chercher des associés qui 
cautionnaient les initiatives : le directeur de la troupe musicale de Mondragón, le pharmacien, notable du village, les différents agriculteurs. En moins de six mois, selon Ormaetxea, il y avait 130 associés potentiels. $90 \%$ de ces associés ont rejoint par la suite de manières directe ou directe les coopératives de sorte qu'on pouvait dire : "Tous ceux qui travaillent sont associés et seuls sont associés ceux qui travaillent », la devise de Mondragón.

La banque, créée en 1959, a collecté auprès d'épargnants des sommes considérables, environ 15 millions de francs en 1980, soit environ 300000 comptes d'épargne, rémunérés à un taux très bas, d'environ 1 à $3 \%$ au début des années soixante selon qu'il s'agit de comptes courants ou de livrets bloqués alors que les taux de crédit accordés aux coopératives varient de 9 à $13 \%$. Ce type d'établissement bancaire intégré a été la clé de réussite du système de Mondragón car il s'agit, au moins durant la première période, de la fonction financement-investissement-budget de chaque coopérative qui est gérée par la CLP.

Durant la décennie, Ulgor et Arrasate font avoir une très forte croissance, profitant des mesures protectionnistes de la dictature espagnole pour conquérir le marché espagnol. Copreci, Ederlan et Lana naissent sur le chemin tracé par les coopératives pilotes. Mais déjà, il est dit que si l'on souhaite que le mouvement coopératif ne soit pas que passager, il faut qu'il s'enracine dans l'éducation et dans les relations économiques et sociales. En 1960, l'école professionnelle dépasse les 300 élèves (contre 21 en 1944).

En 1964, est créé El Grupo Ularco. Avant cette date, la coopérative-mère Ulgor était découpée en départements : fonderie, pièces mécaniques, electro-ménagers, grosses machines, etc. Chaque département va prendre la forme coopérative, avec sa propre indépendance juridique. S'ajoutent deux autres coopératives, Aurki, coopérative $\mathrm{d}$ 'automatismes et Servicio de Ingenieria, coopérative de ventes de technologie. Le groupe Ularco est après la création de la première coopérative en 1955 et la création de la Caja Laboral en 1958, la troisième innovation d'importance de cette première période. Ularco réunit 8 coopératives de 6569 salariés dont 3556 pour Ulgor.

En 1970, El Groupo Ularco est une association qui fédère 40 coopératives dont le chiffre d'affaires est évalué à 7059 millions de pesetas, dont les exportations représentent moins de $10 \%$ et où les dépôts de Caja Laboral représentent $50 \%$ du chiffre d'affaires total. La coopérative le plus importante de Ularco est UGOR (appareils ménagers) mais on note à cette époque une très forte croissance de Fagolectro (composants électroniques)

L'évolution de l'emploi est fulgurante : en 1960, le groupe occupait 479 emplois; en 1965, il comptait 4211 salariés et en 1970, 8743 emplois.

1971-1990. Deux décès symboliques : Franco et Arizmendiarrieta

Cette période est marquée par deux décès qui symbolisent les ruptures par rapport à la période précédente.

Le premier décès est celui du général Franco en 1975 qui va signifier la fin du protectionnisme espagnol. Les coopératives se lancent dans l'ouverture vers les marchés extérieurs au pays basque (en 1990, le poids des exportations représente $15 \%$ du chiffre d'affaires) par l'implantation de firmes à Madrid et à Barcelone puis au Mexique et en Thaïlande. Par ailleurs, s'ouvre en 1981, à Vitoria le premier hypermarché. Plus de 50 nouvelles coopératives sont créées entre 1972 et 1985.

La crise économique mondiale et particulièrement industrielle provoque un désemploi important, de l'ordre de $20 \%$ des effectifs au pays basque, voire plus dans la province de Bilbao. Le point le plus haut de la crise est l'année 1983 mais déjà en 1974, certaines coopératives sont en grande difficulté. Le rôle de la Caja Laboral va être ici essentiel.

Le deuxième décès est celui de son fondateur, Don José Maria Arizmendiarietta ${ }^{7}$ qui meurt en 1976, un an après celle du dictateur espagnol et qui symbolise l'effondrement du mouvement social catholique dans les pays du Nord. Il révèle le recul du sentiment religieux à la fin des années soixante presque partout en Europe, qui est encore manifeste en Espagne, alors qu'il ne 
l'est plus en Italie qui bénéficie d'un regain, moindre en France. Le complexe coopératif va chercher à rationaliser ces pratiques et met en place toute une série d'indicateurs et d'instruments de gestion d'entreprises compétitives. Par exemple, la capitalisation des résultats, une péréquation des résultats avec un fond de solidarité inter coopératives, la flexibilité des calendriers de travail au semestre, la polyvalence des fonctions et des emplois afin d'équilibrer les postes entre les coopératives en excès et celles qui sont en demande d'emplois.

Ce sont ces deux morts symboliques qui ouvrent une nouvelle période. C'est en 1982, que le collectif des salariés-associés décide de limiter leur progression quantitative pour réduire l'influence du coût du personnel sur les ventes. Si le groupe ne licencie pas (interdit par les statuts), c'est au prix d'une diminution du salaire réel, c'est-à-dire une augmentation de la quantité de travail sans accroissement de salaire, une saisonnalisation du travail, des préretraites et un accroissement de la productivité.

La question du sous-emploi se règle en deux étapes. Tout d'abord, les emplois en surnombre dans le domaine industriel sont compensés par les coopératives de distribution. Après cette première compensation, il restait une centaine de salariés sans activité mais rémunéré. Après quelque mise en préretraite, il est décidé de rémunérer les sans emplois tous en formation à hauteur de $20 \%$ sur les salaires des autres «socios». Le mécanisme a pour but de tout faire pour ne pas exclure les travailleurs les moins performants. En faisant peser sur les salaires des travailleurs le poids des sanstravail, on lutte pour leur réintégration car il devient économiquement plus intéressant de les intégrer que de les avoir à charge. Par ailleurs, pour aller dans le même sens, les titulaires de pension ont une représentation à l'Assemblée Générale.

\section{A partir de 1991, MCC ou le pragmatisme organisationnel}

Après une présentation factuelle, on cherchera à répondre aux questions que pose cette stratégie expansionniste puis à évoquer la question sociale.
Le processus engagé dans la période précédente se confirme nettement avec une accélération des exportations par rapport au volume du chiffre d'affaires. En 2002, le poids de l'exportation rapportée au chiffre d'affaires est de $27 \%$.

Au niveau local, il n'y a plus de priorité basque dans la recherche des fournisseurs mais seulement les règles du marché - le prix, la qualité, le service-, ce qui pousse au professionnalisme des coopératives.

Du point de vue de la structure interne, la recherche appliquée et l'innovation est au centre du dispositif du groupe qui est divisé en trois secteurs :

- un secteur industriel divisé en 7 branches rassemblant 70 coopératives occupent 18 000 postes de travail,

- un secteur de distribution mobilise 13000 socios.

- un secteur financier (banque, leasing, assurance) emploie 1500 personnes.

Le secteur industriel est divisé en sept coopératives: automation-automobile, composants, construction, équipement industriel, foyer, biens d'équipement et machine-outil.

Dans chaque coopérative, les travailleurs-associés, rassemblés en assemblée générale, élisent un conseil d'administration, appelé le conseil recteur. Le conseil recteur élit son directeur sur proposition du Président, élu pour 4 ans. Le Conseil Social est représenté uniquement de travailleurs-associés.

Au niveau de MCC, les 7 directeurs de coopératives industrielles, le directeur du secteur financier et le directeur de la distribution siègent au Conseil Général. Ce conseil a une fonction de gestion et de proposition.

La Commission permanente est composée de 16 personnes élues (toutes différentes du Conseil Général) représentant les 9 divisions en pourcentage du nombre de socios, avec une limite de $25 \%$ du total des membres pour une entité. Cette commission permanente décide en soumettant ses choix au Congrès, organe suprême qui regroupe 650 délégués, proportionnellement au nombre de socios, avec la même limite des $25 \%$ de la Commission permanente.

Le Conseil Général est le conseil exécutif de MCC. Si cette structure voit le 
jour en 1991 lors du troisième congrès, l'idée est apparue en 1984, après la violente crise industrielle de 1981-1983.

Concernant les centres de formation, le caractère coopératif est double : d'abord, il s'affirme dans les décisions où siègent trois collèges: le personnel enseignant et administratif, les représentants des parents et enfants et les collaborateurs extérieurs ; ensuite, sur le financement, $10 \%$ est pris sur les excédents des coopératives et le reste est financé par un jeu de bourse.

Concernant les règles de répartition, après $10 \%$ versés en impôt et la dotation à la réserve qui est le double d'une société privée (contre 32,5 \% d'impôt pour une entreprises privée espagnole), les coopératives versent $10 \%$ à l'éducation et 20 $\%$ au titre des réserves pour les coopératives en difficulté (fond inter-coopératif). Le reste est versés aux associés après avoir déduit les avances. Mais ce reversement n'est pas disponible avant le départ de la coopérative.

Chaque entité élargit sa sphère d'influence. Par exemple, sur 1000000 de clients de Caja Laboral, il n'y a plus que 30 000 socios des coopératives; en termes d'investissement, les coopératives ne représentent plus que $5 \%$ des investissements totaux, $40 \%$ représentent les prêts aux particuliers et $35 \%$ constituent des réserves. Il est très important dans l'esprit de Mondragón que l'entraide entre coopératives ne se transforme pas en dépendance.

\section{Les Corporations de Développement Economique Communautaire (CDEC à Montréal}

A partir des années soixante, l'église catholique au Québec s' effondre, comme nous venons de l'évoquer pour Mondragón et pour toute l'Europe. Son importance se consume mais on voit apparaître, sur ces cendres, l'éclosion du mouvement communautaire. Il prend la forme des comités de citoyens, divisés en sous-comités, à l'échelle de la paroisse : lutte pour l'avortement, droit des femmes, revendication de droits sociaux, logement, santé, etc. Autrement dit, les mouvements de contestation de la fin des années soixante occupent le découpage de l'espace physique des pratiques religieuses.
Autour des années soixante-dix, se mettent en place des formes d'organisation autonome : les cliniques communautaires, les carrefours des jeunes travailleurs, les maison de chômeurs. "Les cliniques populaires montrent bien comment les groupes de services remettent en cause les services comme les dispense l'Etat, à savoir la gestion bureaucratique et centralisée sur lesquelles les usagers n'ont aucune emprise, l'organisation du travail où même les professionnels sont contraints à des tâches d'exécution décomposées en éléments parcellisés et routinisés, sans oublier les contraintes et les limites du modèle bio-médical. Les cliniques cherchent ainsi à renouveler aussi bien les contraintes professionnelles que les pratiques de consommation: dans le premier cas, elles le font par la promotion du travail en équipe interdisciplinaire et par une approche soucieuse de répondre aux besoins de la communauté ; dans le second cas, elles y tendent au travers de la cogestion usagerstravailleurs et diverses initiatives pour dépasser la consommation individuelle et passive $» .8$

Dans les années quatre-vingt, on ne revendique plus de droits car non seulement ils ne progressent plus mais la situation économique tend à les restreindre. Le mouvement contestataire est pressé d' intervenir dans le domaine économique. C'est là la grande nouveauté.

Au milieu des années quatre-vingt, on estime à environ 12000 le nombre d'organismes communautaires au Canada dont 8000 pour le seul Québec: des coopératives d'habitation, des groupes d'éducation populaire, des garderies, des maisons de jeunes, des centres de femmes, des coopératives de travail. C'est de là que naissent pour l'essentiel les CDEC.

Les CDEC sont des mouvements nés d'une réaction aux stratégies de croissance pensées par le «haut». En cela, ils intègrent le développement économique et social qui résulte de la volonté des populations de lutter contre l'exclusion, la dégradation de l'environnement et la dévalorisation des territoires. Ils peuvent être interprétées comme une des variantes issues des Community Economic Development des quartiers noirs américains, dans les années soixante. Même si les militants de base n'avaient pas de relais avec ces expériences, 
quelques missions, notamment celle de Boston ${ }^{9}$ rendaient ces expériences connues aux leaders québécois. Mais alors que dans le premier cas, cette prise en charge pouvait prendre une forme coopérative, elle devient au Québec un instrument du développement local alors que ce même mouvement a donné lieu à des formes privatives, les gated communauty aux Etats-Unis donnant en France la forme des résidences sécurisées ${ }^{10}$. Mais cette intreprétation est sujette à caution par ceux qui voient l'origine de l'expérience québécoise dans celle de la Cape Breton Association for Co-op development qui voulait créer une famille d'entreprises coopératives pour le bien-être de la société (CABAJ, 2004).

Toujours est-il que les CDEC sont nés en 1984. Ils sont en fait l'illustration d'un partenariat entre les groupes communautaires, les patronats publics et privés et les syndicats en vue d'une " gouvernance ${ }^{11}$ locale : aménagement et requalification du territoire, lutte contre le décrochage des jeunes, création d'entreprises.

Concrètement, la composition d'un CA d'une CDEC est illustratif :

- 4 représentants d'entreprises privées du territoire ;

- 4 représentants des groupes communautaires (associatifs) ;

- 2 membres des syndicats ;

- 4 personnalités qualifiées;

- 2 représentants d'institution.

Ce CA représente une assemblée générale de 150 à 300 personnes environ sur un territoire variant de 70000 à 140000 habitants.

Les CDEC symbolisent la volonté de revitalisation d'habitants d'une «communauté locale» en favorisant l'insertion par le travail à travers la gestion participative des activités publiques ou privées d'un territoire. Selon J Fontan, ${ }^{12}$ « les CDEC sont nés dans un climat marqué par deux évènements : les restructurations industrielles et l'échec des élections pour l'indépendance du Québec ». En effet, le contexte dans lequel se développent ces initiatives est celui de la désindustrialisation où certains quartiers ont près de $20 \%$ de chômage (de 10, 2 \% en 1981 à 14,7 \% en 1991 puis 16,7 \% en 1997), doublé d'une crise politique (échec référendaire et échec de la gauche). Ce dernier argument peut recevoir deux interprétations différentes: soit, on n'y a pas réussi par le haut, alors on change de stratégie avec la même finalité ; soit on change de finalité, on laisse de côté l'indépendance mais on cherche un système de représentation qui fasse moins appel aux groupes de pressions, aux médiateurs et davantage à la volonté locale. Evidemment l'alternative peut converger en pensant qu'avec un bon système de représentation, on peut parvenir à éclairer la population vers le bon choix de l'indépendance !

Dans les quartiers les plus touchés, c'està-dire les plus pauvres, très francophone, se met en place une résistance à un certain fatalisme. La question de la langue est importante: s'il ne manque pas de voix pour dire que la richesse du Québec provient de son cosmopolitisme et de son bi-linguisme ${ }^{13}$, la communauté francophone insiste pour rappeler que l'innovation sociale est née chez elle. Si on s'accorde à penser que la culture québécoise est fondamentalement américaine, il n'est pas surprenant que les mouvements de résistance proviennent plutôt de la culture minoritaire francophone ${ }^{14}$.

Les trois étapes historiques des CDEC.

Les CDEC reposent sur deux dimensions essentielles : la lutte contre l'exclusion et la défense d'un territoire par sa revitalisation. La proximité avec l'économie sociale est donc contenue dans les prémisses, même si l'activité des CDEC ne se limite pas à elle.

On peut distinguer trois grandes étapes.

A l'initiative de deux intervenantes de la clinique populaire de Pointe Saint-Charles, une tentative $d^{\prime}$ insertion sociale pour la création d'emploi vient buter sur l'inadéquation du projet local d'ateliers aux programmes gouvernementaux. Cet échec, au bout de trois mois d'existence, donne lieu à un rapport d'où sont issues les trois premières CDEC, créés en 1984 et 1985 à Montréal dans trois quartiers, Pointe SaintCharles, Centre-Sud et HochelagaMaisonneuve. Ces CDEC ont pour vocation de mettre en place un nouveau modèle de développement local en partenariat avec l'Etat et les entreprises privées pour répondre à des problèmes urgents. Il est décidé la constitution d'une aide au fonctionnement de 
200000 \$ canadien par an, et d'un fond de soutien aux entreprises et d'investissement de 100000 \$ pour 15 mois. Il était prévu que ce soutien par l’Etat serait dégressif pour pousser les CDEC à s'autofinancer.

Deux missions leur étaient confiées : améliorer l'employabilité et favoriser l'entrepreneuriat dans un territoire défini.

Ces trois premières CDEC sont toujours aujourd'hui les plus importantes en terme d'emplois et de budget et sont regroupés au sein du RESO (Regroupement pour la relance du Sud-Ouest).

La deuxième génération nait en 1987, 1988 et 1989 en étendant l'expérience-pilote à trois autres CDEC. Cette deuxième phase voit l'élargissement à la CDEC du Grand Plateau, du Centre-Nord et Rosemont-Petite Patrie. En 1990, la troisième génération de deux nouvelles CDEC apparaissent, celle de Côte-des-neiges/Notre Dame et Ahuntsic/ Cartierville.

Durant cette troisième phase, le territoire des CDEC est modifié et se superpose sur 7 arrondissements en vue de la gestion de programmes municipaux. Avec une dotation d'environ 900000 \$ par CDEC, on est loin des 200000 pour la première étape. Dans cette phase, la création d'entreprise prend de moins en moins d'importance au profit de programmes définis par le gouvernement. Chaque CDEC fonctionne alors avec une double structure, associative et entrepreneuriale qui assure les deux missions principales : la première porte sur l'employabilité, c'est-à-dire l'information, l'aide à la recherche d'emplois et la formation ; la deuxième a trait à l'entrepreneuriat, c'està-dire le conseil, au montage financier et au financement d'activités nouvelles.

L'étape ultérieure apparaît en 1997 avec la mise en place de 56 centres locaux de développement (CDC) pour le développement local et 150 centres locaux d'emplois (dotés d'un budget de 14 millions de dollars pour Montréal) à travers tout le Québec et dont la gestion vient s'empiler sur les fonctions des premières CDEC.

Sept CDEC regroupés dans l'InterCDEC ont affirmé leur antériorité et le désir d'être accrédité comme CDC et centres locaux en souscrivant aux régles de l'Etat mais aussi en reprenant les acquis des CDEC.
Les trois CDEC les plus avancés sont aussi les plus anciennes: l'Est, le sud-ouest et le centre-sud (qui est le moins industrialis).

Parallèlement, c'est en 1998 que s'ouvre le chantier de l'économie sociale, suite au sommet sur l'emploi tenu en 1996.

Chaque CDEC semble libre de son organisation, de ses priorités en matière de développement économique et des priorités vis-à-vis de l'économie sociale. L'enveloppe budgétaire est divisée en trois volets (fonctionnement, fonds d'investissement et développement de l'économie sociale) actée par une entente de gestion avec les municipalités et le ministre responsable.

Pour fixer un ordre d'idée, à Montréal, les CDEC emploient plus de 200 personnes à Montréal (variant d'1 personne à 45 selon les cas) gèrent un peu plus de 12 millions de dollars canadien en fond d'investissement, 5 millions de RESO Investissements, 2,3 millions de l'économie sociale.

La population couverte par les CDEC varie de 68755 habitants pour le RESO (sudouest de Montréal) jusqu'à 158930 habitants pour la Côte des Neiges. 11 CDEC découpent Montréal et 17 CDEC ont été créés pour le Québec, ce qui signifie que tout le territoire québécois n'est pas encore couvert.

\section{La Banca Popolare Etica}

Les premières expériences de finance éthique ont été menées par les coopératives MAG (Mutuelles d'autogestion) dans les années soixante-dix. Les MAG sont des coopératives financières qui cherchent à créer des circuits de financement alternatifs c'està-dire en direction du social, de l'environnement, de la culture et de l'environnement. Elles s'appiuent sur du capital "indépendant" pour pallier au manque de financement accessible au milieu associatif et à l'économie sociale. Dans son objectif et dans le paysage français, elles ressemblent à la NEF (Nouvelle Economie Fraternelle).

Dans les années quatre-vingt dix, l'adoption d'un texte unique sur les activités bancaires et de crédit prive les coopératives ayant ce type d'activité du droit de collecter l'épargne des personnes physiques. Il faut alors s'organiser pour créer au niveau national une structure capable de collecter et 
distribuer "l'épargne alternative". Par ailleurs, la société civile fait pression pour une activité financière soucieuse de servir le développement humain. C'est dans ce but qu'est créée en 1994 l'association "Verso la Banca Etica" (Vers la banque éthique), qui changera de statut l'année suivante pour devenir une coopérative afin de souscrire le capital social nécessaire à la création de la Banca Etica. Au départ, elle comprend 22 organisations issues du monde associatif, des coopératives ou de la finance solidaire, parmi lesquelles on peut noter le consortium national des coopératives sociales (CGM) ou l'Association italienne d'agriculture biologique, ou encore Caritas Italia qui se réunissent dans le but de créer une "banque de crédit coopératif". Mais dés 1996, c'est le statut de banque populaire qui est finalement retenu. Moins restrictif, il permet de développer l'exercice de l'activité de crédit sur tout le territoire tout en gardant le statut coopératif. En 1997, avec 6 milliards d'euros de capital social, la coopérative commence à acquérir une visibilité nationale. En 1998 les 12,5 milliards sont atteints. Le premier guichet de la Banca Popolare Etica (BPE) est ouvert à Padoue le 8 mars 1999, il y a donc six ans.

La BPE est le produit de deux mouvements :

- un mouvement d'investissement dans le troisième secteur défini exclusivement par le «non-profit». Le secteur a besoin de crédit qu'il ne trouve pas dans les banques traditionnelles. Par exemple, les coopératives ou les associations obtiennent des marchés publics pour la gestion de crèches sans qu'il puisse y avoir de concours des banques qui rechignent à supplanter la lenteur des financements publics. Ce type de demande est assez semblable à celle des commerçants ou des artisans du début du siècle qui fondaient des caisses.

- Un mouvement d'épargnants qui cherche à savoir ce qu'on fait de leur fond, dans un souci de transparence et une volonté de développement local. A la BPE, l'épargnant a quatre possibilités : la première est le moyen de paiement $d u$ compte courant, la carte bancaire (Affinity Card) ; la deuxième est le certificat de dépôts ou dédiés qui finance un projet particulier avec un taux de rémunération de l'épargne choisi par l'épargnant entre 1 et $2 \%$; le troisième est une obligation à taux fixe, garanti et soumis à moins d'imposition fiscale; le quatrième est proposé à une société de gestion de l'épargne avec un taux d'intérêt plus élevé (les Fonds Communs Etica Sgr).

Les deux mouvements ne sont pas totalement symétriques et il y a des arbitrages à faire entre les secteurs choisis par les épargnants qui ne sont pas nécessairement les choix des investissements; par exemple, la coopération internationale est choisie par $49 \%$ des épargnants alors que ce secteur n'est financé qu'à hauteur de $14 \%$; inversement, la coopération sociale est choisie par $17 \%$ des épargnants alors qu' elle est financée à hauteur de $45 \%$ du total des crédits.

La BPE repose sur trois niveaux de décision :

- Le premier niveau repose sur un réseau de relations, une connaissance approfondie du troisième secteur de membres de la BPE par lesquels doit passer obligatoirement le projet d'investissement : le réseau Caritas (il existe 30 associations diocésaines) par exemple est un des plus connus mais on peut également citer 300 paroisses, 7 régions, 20 provinces qui représentent les personnes juridiques (16 $\%$ du total, $84 \%$ représentant des personnes physiques).

- Le deuxième niveau est un réseau de volontaires : 20500 personnes physiques bénévoles et 3300 organisations composent la banque qui ont acheté au moins 5 parts de 51 euro chacune. Lorsque ces volontaires forment un groupe de 200 actionnaires, ils peuvent former une circonscription locale. Il est alors nommé un coordonnateur local qui examine le caractère éthique du projet. Il existe 70 circonscriptions locales sur tout le territoire italien qui comprend 21 régions, dont 1 en Calabre et 3 en Toscane.

- Le troisième niveau est celui de l'étude financière sur la faisabilité du projet. Il est le fait de 8 succursales (Padoue qui est le siège,Trévise, Milan, Bologne, Brescia, Florence, Venise et Rome). Un fond de péréquation a été créé pour permettrent avec 3 à $4 \%$ des dépôts de financer $15 \%$ d'investissement en Sicile. 
Quelles sont les garanties mises en place par le BPE pour ne pas trahir le projet?

A priori, on pourrait répondre qu'il n'existe aucune garantie pour ne pas trahir des projets initiaux! Mais disons qu'il y a deux remparts importants. Tout d'abord, le projet peut-être porté par un évêque ou un membre du PCI mais il ne peut le faire qu'au travers de la médiation d'une structure sociale du tiers secteur, par exemple une coopérative de détenus. La BPE ne relève donc pas du microcrédit mais de la finance éthique ${ }^{15}$ car elle ne prête qu'exceptionnellement à des individus (lors d'un prêt pour un achat d'un premier appartement ou pour une adoption d'enfants ) mais en général, ce sont des prêts à des coopératives ou des associations (le nonprofit) ou à des entreprises privatives lorsqu'elles portent un projet social, biologique ou culturel.

Ensuite, le rôle de la circonscription est essentiel car il s'agit d'un tri de projets fondé sur la connaissance d'un territoire réduit.

La BPE croise donc trois types d'acteurs : les actionnaires épargnants, les médiateurs du tiers-secteur et la structure opérationnelle de la BPE. Lorsque les médiateurs proposent le projet d'une coopérative sociale, il se peut que celle-ci ait obtenu une mission de l'Etat. Ce qui lie ces trois types d'acteurs, c'est une philosophie en action.

Aujourd'hui, l'épargne est abondante, environ 316000000 d'euros contre 200000 000 d'investissement. D'où le fait que la BPE cherche de nouveaux marchés à travers les marchés biologiques. Vingt et un ambulants se déplacent sur tout le territoire, découpé en quatre zones. Ils perçoivent un salaire fixe qui permet d'éviter des abus commerciaux. Par l'intermédiaire des structures sociales, Les taux sont identiques sur tout le territoire, environ 1 point au-dessus des autres banques dans le Nord et 2 ou 3 points plus bas dans le Sud. A long terme, les taux moyens sont de plus $1 \%$ du EURIBOR.

La BPE a activé tous les services d'une banque virtuelle car tous les comptes courants sont gérés par internet. Aujourd'hui, BPE est une société coopérative par action à responsabilité limitée dont l'objet est selon l'article 4 de ses statuts « la récolte de l'épargne et l'exercice du crédit».
Elle définit ainsi dans l'article 5 de ses statuts ce qu'est En 2002, Fabio Salviato, Président de la $\mathrm{BPE}$, pouvait prétendre à un taux de croissance de $100 \%$ sur 3 années consécutives, et d'un taux de perte de 1,5\% largement inférieur au 4,5\% de moyenne pour les banques italiennes. La même année, sur 18000 membres, 2700 étaient des "personnes juridiques" (dont plus de 300 collectivités territoriales: 300 municipalités, 20 provinces et 7 régions). 160000000 Euros ont été collectés, le capital souscrit était de 12600000 euros et 8000000 ont été affectés aux financements de projets.

La BPE n'a pas ouvert de guichets sur tout le territoire : 1 Italie a été divisée en quatre : Nord-Ouest, centre, sud et Nord-est. Ce dernier représente 7413 actionnaires, quatre agences et quatre banquiers ambulants. La BPE est présente dans chaque province italienne, par le biais des "groupes locaux d'actionnaires", réunis dans le "réseau des actionnaires". Ce réseau se veut un des moyens de la politique de transparence et de participation prônée par la banque. Il s'appuie sur les "Groupe d'Initiative Territoriales" mis en place par "Verso la Banca Etica" pour appuyer la collecte des fonds nécessaires à la création de la BPE.

Le département "Recherche » est devenu une fondation indépendante, la Fondazione Culturale Responsabilita Etica Onlus ${ }^{16}$ : elle a mis en place la méthode d'évaluation VARI, qui prend en compte la participation, la transparence, l'égalité des chances, la valeur sociale produite, les retombées écologiques, la place du bénévolat, l'implication dans le territoire, en quelque sorte des critères d'évaluation sociale des projets. En dehors de proposer aux épargnants le choix du secteur où leur épargne sera investie (environnement, coopération sociale, coopération internationale, qualité de la vie), la BPE a mis en place les "titres éthiques", destinés à financer les organismes d'économie sociale. Des avantages fiscaux importants sont consentis pour les investisseurs qui les acquièrent. Ces fonds sont gérés par Etica Sgr (Società di Gestione del Risparmio), fondé en 2001 en collaboration avec la Banque Populaire de Milan et dont BPE détient 51\% des part et assure l'aspect développement stratégique (la BPM s'occupant de la gestion 
technique). Par cette intervention, la BPE va mettre fin à la particularité de l'Italie en Europe qui est de posséder un en-cours de fonds éthique important (environ 1800 millions d'euro), ce qui place l'Italie au double de la France concentré sur deux seuls fonds ; la banca Etica se lance donc sur ce marché occupé jusque-là que par l'Eglise catholique.

Deux expériences sont intéressantes et permettent d'éclairer les actions de la Banca Etica. La première a trait au principe des bons dédiés. L'épargnant souscrit au financement d'un projet. Par exemple, à Padoue, la Fondation La Casa souhaitait financer des habitations pour les immigrants. La BPE a créé des bons dédiés pour cette opération et la Fondation a reçu un crédit de 3,5\% pour construire ces habitations dont les principaux épargnants qui ont reçu un taux de $0 \%$ sont les entrepreneurs des entreprises employeurs de cette main d'oeuvre.

Le deuxième exemple est celui de Posto delle Fragole, coopérative créée à la fin des années soixante-dix à Trieste. A cet endroit, résidait un hôpital psychiatrique recevant des personnes atteintes de graves maladies. A partir de 1971, trois mouvements vont transformer la structure asilaire : la fermeture de l'Hôpital Psychiatrique, la construction d'un réseau de services alternatifs et l'avancée des théories psychiatriques reconnaissant la porosité des frontières entre malade et non-malade et la co-construction de parcours thérapeutiques fondées sur une participation active des tous les corps de métiers. C'est dans ce contexte qu'est créée la coopérative d'Il Posto delle Fragole qui gère un bar, un restaurant et un hôtel animés par des patients, des médecins et tous les autres corps.

D'autres exemples pourraient encore illustrer notre propos : celui par exemple de la Citta Futura qui grâce au financement de la BPE a reconstruit 11 maisons pour les transformer en Hôtel et faire revivre le petit village de Riace en Reggio Calabria ou encore l'intervention massive de la BPE en faveur de deux coopératives agricoles d'une communauté religieuse des moines de Lanuvio dont le redressement comptable a été largement dû aux certificats de récolte mis en place par la BPE.

\section{Conclusion}

Dans les représentations traditionnelles utilisées en économie, le transfert technologique exprime que les sources de l'innovation au Nord sont transférées avec plus ou moins de succès au sud. Pourtant, la conjugaison originale de solidarités traditionnelles et de solidarités nouvelles tend à montrer que l'innovation sociale apparaît dans les couches vulnérables au Nord comme au Sud. On perd ainsi l'exclusivité géographique des sources de l'innovation technologique défendue par les théoriciens de l'économie néo-classique. Il faut alors de manière pragmatique et fine étudier ces différentes expériences pour en tirer des ingrédients :

Chaque cas apparaît sous la pression d'une nécessité sociale liée à un chômage élevé. Mais le mouvement social ne se réduit pas à une "nécessité sociale » car il intègre des dimensions non économiques. A ce titre, il ne suffit pas qu'il y ait un intérêt commun pour qu'une population s'engage dans une action collective car cet engagement suppose un coût de participation. Or, beaucoup peuvent préférer user de la position du passager clandestin. A ce niveau, les mouvements alter mondialistes sont très instructifs car la manière dont ils usent des technologies de l'information pour créer une occupation de l'espace public, de masse à des heures intersticielles est révélateur de nouvelles formes de luttes sociales.

1. Dans chaque cas, la société civile inscrit son action dans un berceau historique qui lui est favorable: la pression de Franco et de la guerre civile sur le pays basque, l'éclatement de la présence écclésiastique au Québec, la poussée ultra-gauche en Italie.

2. Un référent commun sert de lien : la langue basque, le français au Québec.

3. Un leader charismatique se détache assez rapidement: Arizmendiaretta au Pays basque ou N. Neamtam pour l'économie sociale ou C.Yaccatini pour les CDEC au Québec. La difficulté importante est la manière dont on poursuit l'œuvre d'une personnalité charismatique avec les acteurs qui font le processus; autrement dit, une monopolisation trop forte du leader-ship viendrait à nuire au 
mouvement. On a connu le népotisme des coop en France. Le cas de N. Neamtam et C. Yaccatini au Québec sont intéressants parce qu'ils augurent de nouveaux types d'entrepreneuriat social qui ne changent pas d'habit, qui ne "retournent pas leur veste » mais qui sont à la fois des militants et des entrepreneurs.

4. Le secteur industriel n'est pas délaissé : le secteur industriel (Angus, Fagor, ou les coopératives industrielles italiennes) conforte la thèse que nous avons toujours défendu d'un non-dépassement de l'industrie par la société tertiaire.

5 . La règle est la coopération à l'intérieur qui se traduit par une grande implication et la compétition à l'extérieur qui pousse à une démarche entrepreneuriale. Il n'y a donc pas d'isomorphisme du groupe mais au contraire insertion groupée dans la division internationale du travail grace aux technologies de l'information.

6. La formation constitue la base de l'employabilité : CDEC au Québec, école polytechnique au Pays basque.

7. La création d'outils financiers spécifiques : RISQ (Réseau d'Investissement Social du Québec) au Québec, Banca Etica en Italie et Caja Laboral en Espagne.

8. Le rapport d'extériorité à l'Etat est prononcé : vis-à-vis du franquisme, d'un Etat impuissant en Italie et des gouvernements au Québec. C'est ce qui distingue le plus profondément ces expériences d'économie sociale de ce que l'on appelle en France l'économie solidaire qui reste, malgrè les discours sur l'espace public, fortement enracinée dans les relations avec l'Etat.

Des critères d'efficacité sont revendiqués, créant des instruments efficaces $\mathrm{d}^{\prime}$ intercoopération et la redistribution presque totale des ressources acquises. La coopération n'est pas entendue comme une pratique qui peut servir au mieux les intérêts individuels mais comme l'intérêt du collectif.

\section{Notas:}

${ }^{1}$ Cette étude a été réalisée grace à l'appui de l'Institut pour la recherche de la Caisse des Dépôts et Consignations de Paris. Je remercie particulièrement Isabelle Laudier de nous avoir fait confiance.

${ }^{2}$ En 1832, le IV congrès coopératif disait « ...qu'il était unaniment résolu que les coopérateurs, quels qu'ils soient, ne s'identifient à aucun principe, soit religieux, soit irrélégieux, soit politique... ». cité par P. Toucas, 2005.

${ }^{3}$ Usatorre est mort en 1970, Larranaga en décembre 2004 alors que Ortubay a quitté la coopérative 6 mois après sa création.

${ }^{4} \mathrm{~J}$-M Ormaetxea nous a reçus le 22 octobre 2004 à Palacio Otalora, à Mondragón.

${ }^{5}$ L'expression « anticipos laborales », traduite par " avance sur travail », est importante car elle implique que la vraie rémunération du travail se fera postérieurement et qu'il sera toujours possible $\mathrm{d}^{\prime}$ avoir «plus » mais également $\mathrm{d}^{\prime}$ avoir « moins ».

${ }^{6}$ Chiffres de 1970, transformés de pesetas en euro.

${ }^{7}$ Un musée privé à Palacio Otalora lui est dédié d'une simplicité à la hauteur de l'homme.

${ }^{8}$ C. Valadou, B. Rufo, D. Bordeleau et J. Lemire, L'entrepreneurship communautaire, le communautaire et l'économie, alliance contre-nature ou nouvelle avenue, CDEC centre Sud/ Plateau Mont Royal, Ronétypé 1997.

${ }^{9}$ Entretien avec Nancy Neamtan le 21 juin 2004 à Montréal

${ }^{10}$ Une résidence sécurisée est un ensemble de six à huit bâtiments de trois étages, soit environ 200 appartements, clos avec portail d'entrée électronique, souvent équipé d'une piscine, se situant à proximité des entrées des grandes villes, proche des bretelles de périphériques. Destinées à une population mobile, de classe moyenne, souvent jeunes, elles sont l'objet de défiscalisation pour les acquéreurs qui donc, $n^{\prime} y$ résident pas. Un des plus grands constructeurs est l'entreprise toulousaine Monney-Decroix.

11 cette expression anglo-saxonne a été employée par les technocrates embarrassés qui voulaient montrer que le pouvoir pouvait venir d'en bas sans lâcher le haut!

${ }^{12}$ Entretien avec Jean-Marc Fontan le 21 juin 2004

${ }^{13}$ Par exemple, notre collègue Marguerite Mendell de l'université Concordia

${ }^{14}$ « Le Québec est une petite société francophone de 7 millions de personnes...c'est sans doûte en partie à cause de notre réalité de minorité que nous avons dû, dans notre histoire, nous serrer les coudes et nous appuyer sur des solidarités internes pour continuer en tant que peuple à progresser et à nous épanouir ». N.Neamtan, Séminaire Porto Alegre, février 2002

${ }^{16}$ Entretien avec son Président, U. Biggeri, le 18 février 2005 à Rome. 